\title{
Brodifacoum Induces Early Hemoglobinuria and Late Hematuria in Rats: Novel Rapid Biomarkers of Poisoning
}

\author{
Kyle M. Ware ${ }^{a}$ Douglas L. Feinstein ${ }^{d, f}$ Israel Rubinstein ${ }^{e, f}$ Guy Weinberg ${ }^{d, f}$ \\ Brad H. Rovin ${ }^{b}$ Lee Hebert ${ }^{b}$ Navin Muni ${ }^{a}$ Rachel E. Cianciolo ${ }^{c}$ Anjali A. Satoskar ${ }^{a}$ \\ Tibor Nadasdy ${ }^{a}$ Sergey V. Brodsky ${ }^{a}$ \\ Departments of a Pathology, ${ }^{\mathrm{b}}$ Medicine, and ${ }^{\mathrm{C}}$ Veterinary Biosciences, The Ohio State University, Columbus, Ohio, \\ Departments of ${ }^{\mathrm{d}}$ Anesthesiology and ${ }^{\mathrm{e}}$ Medicine, University of Illinois at Chicago, and ${ }^{\mathrm{f}}$ Jesse Brown VA Medical Center, \\ Chicago, III., USA
}

\section{Key Words \\ Brodifacoum · Superwarfarin · Acute kidney injury · \\ Urinalysis · Hematuria · Hemoglobinuria · N-acetylcysteine}

\begin{abstract}
Introduction: Brodifacoum (BDF) is a superwarfarin that is used primarily as a rodenticide. There have been increasing numbers of reports of human cases of accidental or intentional BDF ingestion with high mortality rate. Its broad availability and high lethality suggest that BDF should be considered a potential chemical threat. Currently, there is no biomarker for early detection of BDF ingestion in humans; patients typically present with severe coagulopathy. Since we demonstrated earlier that warfarin can induce acute kidney injury with hematuria, we tested whether BDF would also lead to change in urinary biomarkers. Material and Methods: BDF was administered to Sprague Dawley rats via oral gavage. $\mathrm{N}$-acetylcysteine (NAC) was given per os in drinking water $24 \mathrm{~h}$ prior to BDF. Urinalysis was performed at different times after BDF administration. Anticoagulation and serum creatinine levels were analyzed in the blood. $\boldsymbol{R e}$ sults: We observed that within a few hours the animals developed BDF-dose-dependent transient hemoglobinuria,
\end{abstract}

which ceased within $24 \mathrm{~h}$. This was accompanied by a transient decrease in hematocrit, gross hemolysis and an increase in free hemoglobin in the serum. At later times, animals developed true hematuria with red blood cells in the urine, which was associated with BDF anticoagulation. NAC prevented early hemoglobinuria, but not late hematuria associated with BDF. Conclusions: We propose that transient early hemoglobinuria (associated with oxidative stress) with consecutive late hematuria (associated with anticoagulation) are novel biomarkers of BDF poisoning, and they can be used in clinical setting or in mass casualty with BDF to identify poisoned patients.

๑ 2015 S. Karger AG, Basel

\section{Introduction}

Superwarfarins are chemically modified forms of warfarin with up to 100 times greater potency than warfarin, and they are used primarily as rodenticides. Brodifacoum (BDF) is one of the most commonly used superwarfarins in the USA [1]. The main pathogenic mechanism of BDF action is similar to warfarin, namely a reduction of bioavailable vitamin $\mathrm{K}$ resulting in excessive anticoagulation

\section{KARGER 125}

(c) 2015 S. Karger AG, Base

0250-8095/15/0415-0392\$39.50/0

E-Mail karger@karger.com

www.karger.com/ajn
Sergey V. Brodsky, MD, PhD

Department of Pathology, The Ohio State University

333 W 10th Ave, Graves Hall, B078

Columbus, $\mathrm{OH} 43210$ (USA)

E-Mail sergey.brodsky@ osumc.edu 
and death. BDF follows zero-order elimination pharmacokinetics [2], and it has prolonged half-life elimination from plasma, which was reported to be between 24 and 56 days in humans $[3,4]$, up to 7 days in the serum in rats [5] and up to 92 days in mice [6]. Unlike warfarin, BDF is highly hydrophobic (water solubility is only $3.8 \mu \mathrm{g} / \mathrm{l}$ ), and it contains an organic bromide moiety that could partly underlie its very prolonged duration of toxicity [5].

Since its introduction as a rodenticide in the late 1980s, there have been increasing numbers of patients with accidental or intentional BDF ingestion. Such cases are occurring at a rate of more than 10,000 individuals per year, mostly in children under the age of 6 years, with a mortality rate of about $20 \%[7,8]$. In addition, due to its easy availability, there is a concern that BDF could be used as a chemical weapon in terrorist attacks [5]. Currently, there are no early biomarkers to identify BDF ingestion; patients typically present to a medical facility with already severe anticoagulation. Availability of a biomarker will help in rapid identification of poisoned patients, initiate treatment and reduce the number of casualties. We previously demonstrated that $\mathrm{BDF}$ results in acute kidney injury in animals with chronic kidney disease, but not in controls [9]. Uncontrolled BDF treatment was associated with hematuria, which developed 2-3 days after poisoning and was more significant in rats with chronic kidney disease than in controls [9]. These $\mathrm{BDF}$ renal effects were associated with its anticoagulation properties and were similar to the effects of warfarin on the kidney in humans and rats [9-11]. Accordingly, the aim of this study was to investigate renal effects of BDF administered in a controlled dose in rats and to determine whether $\mathrm{BDF}$ poisoning is associated with early renal damage that can be detected rapidly and non-invasively by first responders before coagulopathy is manifested.

\section{Material and Methods}

\section{Animals}

All experiments were conducted in accordance with the NIH Guide for the Care and Use of Laboratory Animals and approved by the Institutional Animal Care and Use Committee. Adult male Sprague Dawley rats (180-220 g, Harlan Laboratories, Indianapolis, Ind., USA) were provided with food and water ad libitum.

\section{Treatment with BDF and Antioxidant}

BDF (Sigma-Aldrich, St. Louis, Mo., USA) was dissolved at a concentration of $2 \mathrm{mg} / \mathrm{ml}$ in $100 \%$ methanol, then diluted at $1: 1$ ratio with $100 \%$ ethanol, giving a final concentration of fully dissolved $1 \mathrm{mg} / \mathrm{ml} \mathrm{BDF}$. The stock solution was aliquoted and kept at $-20^{\circ} \mathrm{C}$ until use. For oral gavage, the BDF stock solution was dissolved in distilled water to achieve the desired dosage; the total volume for gavage was $0.6 \mathrm{ml}$ per animal.
The antioxidant N-acetylcysteine (NAC; Sigma-Aldrich, St. Louis, Mo., USA) was given per os in drinking water in the concentrations described below. Daily water consumption was measured, and daily NAC dosage was calculated from the animal weight.

\section{Urinalysis}

Free-catch urine was collected. Urinalysis was performed using DiaScreen (Chronimed Inc., Minnetonka, Minn., USA) reagent strips (dipstick) in the urine $[9,11]$. Hematuria was graded using a semi-quantitative scale of $0-3+$. Score 0 was designated for negative hematuria, score $1+$ for mild hematuria, score $2+$ for moderate hematuria and score $3+$ for severe hematuria.

\section{Analyses of Blood Samples}

Blood (approximately $150 \mu \mathrm{l}$ ) was collected from the tail by a small incision. Serum creatinine ( $\mathrm{SCr}$ ) was measured using a creatinine reagent assay (Raichem, San Marcos, Calif., USA) according to the manufacturer's protocol. Briefly, $20 \mu \mathrm{l}$ of serum was mixed with $200 \mu \mathrm{l}$ of working reagent at $37^{\circ} \mathrm{C}$ in a 96 -well plate, and the absorbance was read at $510 \mathrm{~nm}$ at 40 and $100 \mathrm{~s}$ using a Molecular Devices Versa Max plate reader (Molecular Devices, Sunnyvale, Calif., USA). Hemoglobin was measured using a Hemoglobin Assay Kit (Sigma-Aldrich, St. Louis, Mo., USA) according to the manufacturer's protocol. Briefly, $50 \mu$ of plasma was transferred into a well along with $200 \mu \mathrm{l}$ of reagent. A calibrator and blank were run each time. The samples were then incubated for $5 \mathrm{~min}$ at room temperature before measuring the absorbance at $400 \mathrm{~nm}$ using the end point method (Softmax Pro 6.1) on the Molecular Devices Versa Max plate reader (Molecular Devices, Sunnyvale, Calif., USA). Calculations were made comparing the plasma sample minus the blank, against the calibrator minus the blank.

Prothrombin time (PT) was measured using an Electra 750 coagulation analyzer (Medical Laboratory Automation, Pleasantville, N.Y., USA) according to the manufacturer's protocol. Briefly, blood was collected into tubes containing $3.8 \%$ sodium citrate as the anticoagulant in a ratio of 9:1. The blood was centrifuged at $1,000 \mathrm{RCF}$ for $15 \mathrm{~min}$. Then, $0.1 \mathrm{ml}$ of serum was placed in the incubation station for $3 \mathrm{~min}$ and $0.2 \mathrm{ml}$ of warm thromboplastin was added. The pipette plunger was pushed down as the test was started. Clotting time was recorded. We used a 'surrogate' international normalized ratio (sINR) by comparing PT after and before the treatment, as described previously $[9,11]$. The average PT from 100 untreated rats was used as the normal PT time (20.7 s).

Renal morphology was evaluated by 2 independent renal pathologists blinded to the experimental group. Kidneys were cut at the longitudinal axis; a half of each kidney was embedded in paraffin after fixation in $10 \%$ buffered formalin for $24 \mathrm{~h}$. Three micrometer sections were stained with hematoxylin and eosin. Immunohistochemical analysis was performed on sections of paraffin-embedded tissue after antigen retrieval, according to the manufacturer's protocol. Anti-CD31 antibodies (BD Bioscience, San Jose, Calif., USA) were used for endothelial cell staining.

\section{Statistical Analysis}

Results are presented as mean \pm standard deviation if not otherwise specified. Differences between groups were analyzed by the two-paired t test or analysis of variance (ANOVA) test, if applicable. Tukey post hoc test was performed to analyze the differences between groups in conjunction with ANOVA. p values $<0.05$ was considered statistically significant. 


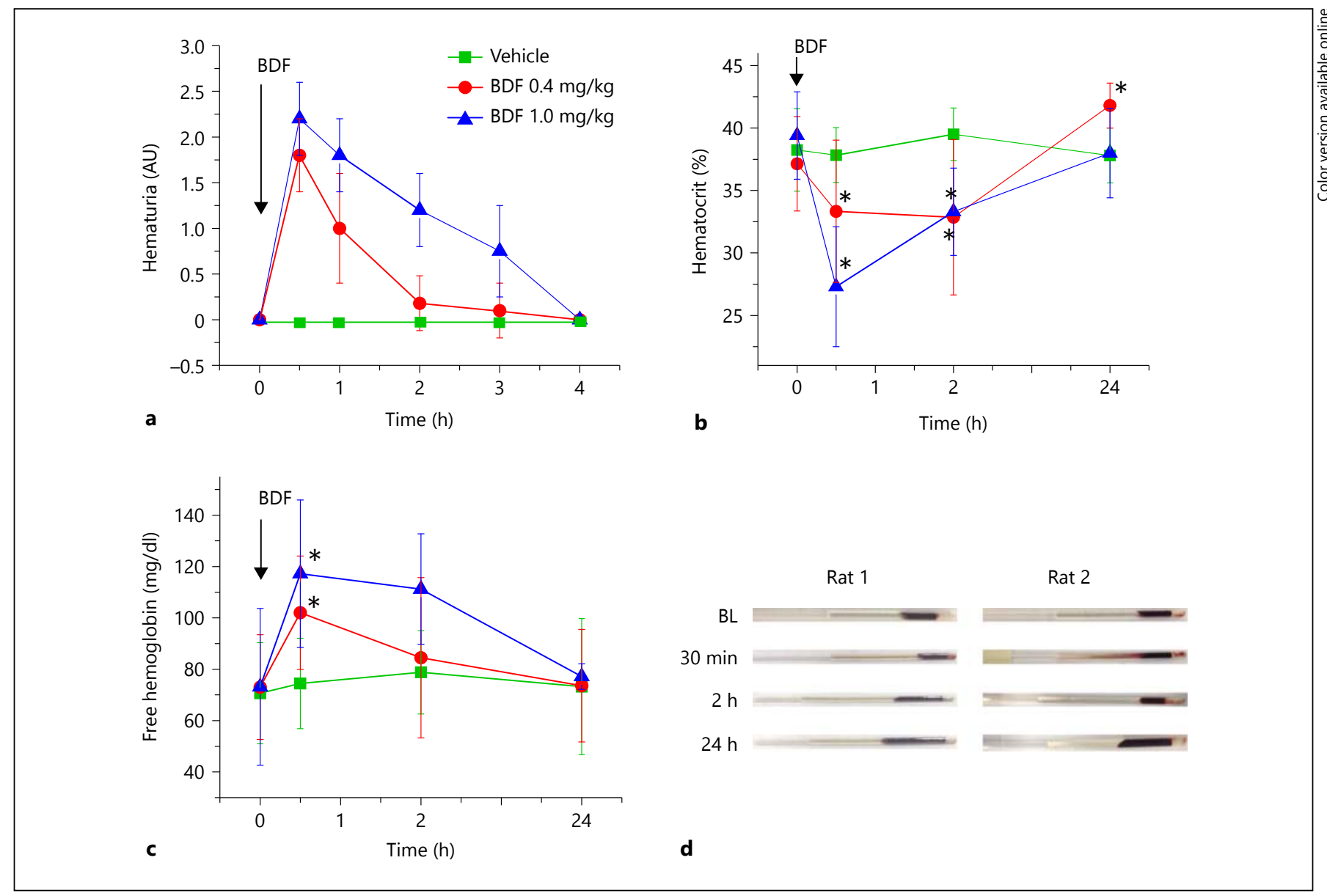

Fig. 1. Early BDF effects on urinalysis and hematocrit. The following number of animals was treated with BDF: $0.4 \mathrm{mg} / \mathrm{kg}, \mathrm{n}=$ $16 ; 1.0 \mathrm{mg} / \mathrm{kg}, \mathrm{n}=6$. Vehicle-treated rats $(\mathrm{n}=15)$ received the same volume of solvent. a A single dose of BDF was administered via oral gavage to rats in the doses shown. Urine was probed with a DiaScreen reagent strip (dipstick) at $30 \mathrm{~min}$ and hourly thereafter up to $4 \mathrm{~h}$ after BDF administration. Results were graded using a semi-quantitative scale of $0-3+$. Score 0 was designated for

\section{Results}

\section{BDF Induces Hemoglobinuria Early after} Administration

After a single gavage with BDF, animals developed early hemoglobinuria/hematuria, which was detected by a DiaScreen reagent strip (dipstick). This BDF-induced hematuria/hemoglobinuria was evident at $30 \mathrm{~min}$ and showed dose-dependency on the amount of BDF administered (fig. 1a). Based on the manufacturer's data, this test is positive for both hematuria and hemoglobinuria. Therefore, we performed a microscopic analysis of the urine sediment and did not find any red blood cells no hematuria, score $1+$ for mild hematuria, score $2+$ for moderate hematuria and score $3+$ for severe hematuria. b Hematocrit was determined in the blood samples at $30 \mathrm{~min}, 2$ and $4 \mathrm{~h}$ after BDF treatment. c Free hemoglobin was measured in the serum using the Hemoglobin Assay Kit. d Gross appearance of blood samples after $0.4 \mathrm{mg} / \mathrm{kg}$ (rat 1) and $1.0 \mathrm{mg} / \mathrm{kg}$ (rat 2) BDF treatment. Gross hemolysis was evident at $30 \mathrm{~min}$ and $2 \mathrm{~h}$ after BDF administration.

(RBCs) in the samples. This suggests that BDF induces rapid hemoglobinuria, and not hematuria.

Next, we analyzed blood samples collected at $30 \mathrm{~min}$, 2 and $24 \mathrm{~h}$ after BDF treatment. There was an initial temporal decrease in hematocrit after BDF administration (fig. 1b). Thus, $30 \mathrm{~min}$ after administration of $0.4 \mathrm{mg} / \mathrm{kg}$ $\mathrm{BDF}$, hematocrit decreased from $37.14 \pm 3.77$ to $33.33 \pm$ $5.69 \%(\mathrm{p}=0.0211)$. After 2 hours, hematocrit was still low (32.86 $\pm 6.21 \%, p=0.0158$ as compared to baseline), but it recovered $24 \mathrm{~h}$ after administration of $0.4 \mathrm{mg} / \mathrm{kg} \mathrm{BDF}$ $(41.80 \pm 1.75 \%, p=0.009$, as compared to baseline). Treatment with a higher dose of BDF $(1.0 \mathrm{mg} / \mathrm{kg})$ resulted in a greater decrease in hematocrit at $30 \mathrm{~min}$ after $\mathrm{BDF}$ 

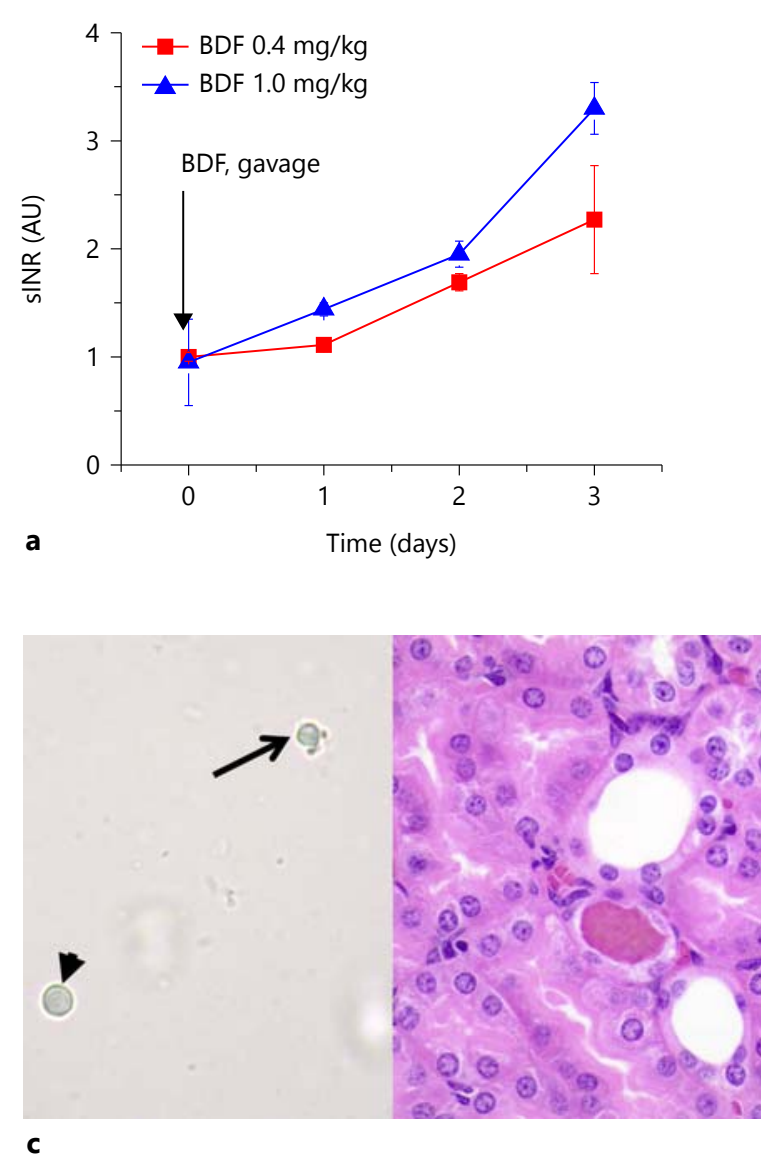
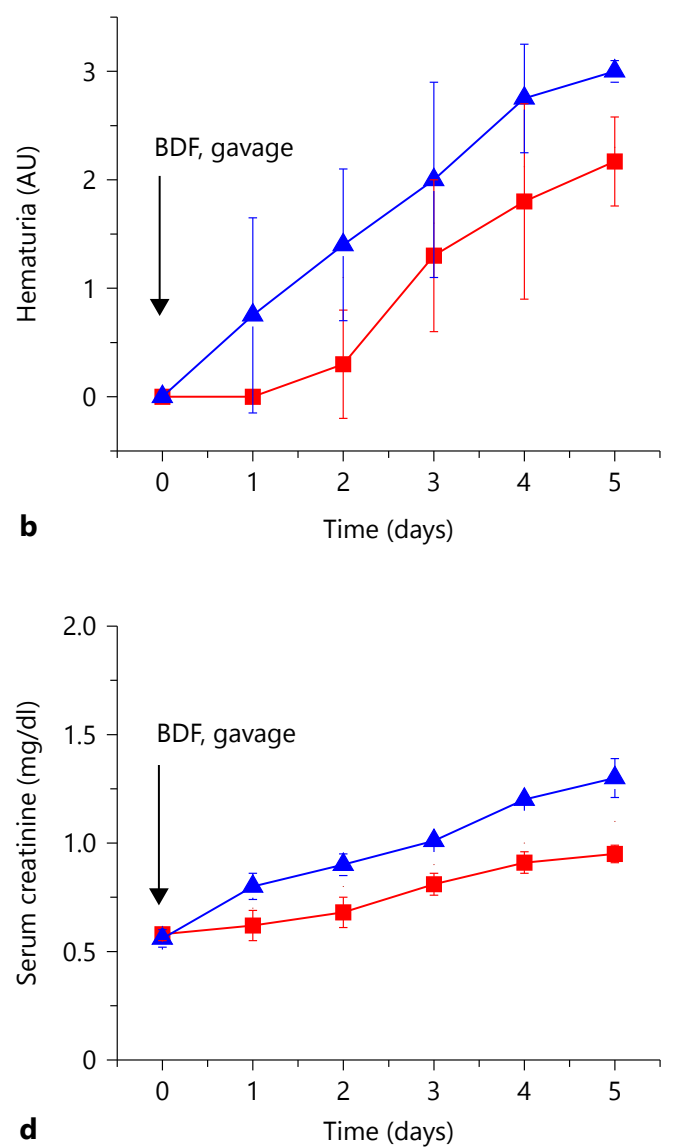

Fig. 2. Late effects of BDF on the renal function and coagulation. The following number of animals was treated with BDF: $0.4 \mathrm{mg} /$ $\mathrm{kg}, \mathrm{n}=16 ; 1.0 \mathrm{mg} / \mathrm{kg}, \mathrm{n}=10$. a PT was measured daily after a single BDF administration via oral gavage. Changes in PT are depicted as sINR as compared to control non-treated rats. b Treatment with BDF resulted in hematuria as detected by DiaScreen reagent strip (dipstick). Urinalysis was performed daily after BDF treatment. c Light microscopy of the urine sediment (left) and a kidney section (right). Urinalysis showed RBCs (ar-

(fig. 1b), but this also recovered at $24 \mathrm{~h}$ after administration ( $38.00 \pm 3.58 \%, \mathrm{p}=0.4474$ as compared to baseline). Grossly, we observed hemolysis in blood samples collected at $30 \mathrm{~min}$ after BDF, but not at 2 or $24 \mathrm{~h}$ (fig. 1d). Measurement of free hemoglobin in the serum showed a dosedependent increase which lasted for at least $2 \mathrm{~h}$ after BDF treatment, but was normalized $24 \mathrm{~h}$ after administration (fig. 1c). These results suggest that that the substance detected by the reagent strip in hematuric/hemoglobinuric urine is hemoglobin, which was consistent with absence of RBCs in the urine. We attempted to measure free hemoglobin in the urine using the same Hemoglobin Assay rowhead) and acanthocytes (arrow) in the urine. A representative photograph of the urine from a rat treated with $0.4 \mathrm{mg} / \mathrm{kg}$ BDF at day 3 after the treatment. Histologically, there was a single RBC cast seen in distal tubule in animals treated with $1.0 \mathrm{mg} / \mathrm{kg}$ BDF. Note mild acute tubular injury with vacuolization of tubular epithelial cells (Hematoxylin and Eosin). Magnification 600x. d $\mathrm{SCr}$ was measured daily after BDF administration in the tail vein (up to day 3 ) and in the postmortem blood (days 4 and 5) after BDF treatment.

Kit, but because this kit is $\mathrm{pH}$ sensitive, our results were inconclusive. Because of the small volume of urine collected, we were not able to normalize $\mathrm{pH}$ in the samples.

\section{Late Renal Effects of BDF Are Associated with \\ Anticoagulation}

The primary action of BDF is anticoagulation. Consistent with this, the PT was significantly and rapidly increased after BDF administration in a dose-dependent manner (fig. 2a). Thus, sINR was increased to $2.27 \pm 0.58$ and $3.31 \pm 0.24$ at day 3 after $0.4 \mathrm{mg} / \mathrm{kg}$ and $1.0 \mathrm{mg} / \mathrm{kg}$ of $\mathrm{BDF}$, respectively (we were able to collect tail blood with- 
out risk of significant bleeding up to day 3 after BDF administration). Simultaneously, there was an increase in hematuria/hemoglobinuria detected by dipsticks, which also was BDF dose-dependent (fig. 2b). Microscopy of the urine sediment showed RBCs in the urine, including acanthocytes, indicating true glomerular hematuria (fig. 2c). Histological evaluation of kidney sections did not show significant pathologic changes, similar to our data with uncontrolled BDF poisoning [9]. There was mild acute tubular injury in proximal tubular epithelial cells with focal loss of brash border and mild vacuolization of proximal tubular epithelial cells (fig. 2c). No tubular or Bowmen's space RBCs were seen. A single RBC tubular cast was seen in an animal treated with $1 \mathrm{mg} / \mathrm{kg}$ BDF (fig. 2c). No RBC tubular casts were seen in any of other kidneys obtained from 22 animals treated with BDF. Immunohistochemical analysis with an antibody to CD31 was performed, highlighting the similar number of capillaries and the pattern in the peritubular capillaries and vasa recta distribution in both BDF and vehicle-treated rats (data not shown).

SCr levels were increased after BDF treatment in a dose-dependent manner (fig. 2d). However, those changes were not statistically significant between groups (ANOVA, $\mathrm{p}=0.2820$ ), which corresponds to our previous data where animals had free access to BDF in pellets [9]. No significant changes in SCr were observed in samples collected at $30 \mathrm{~min}$ or $2 \mathrm{~h}$ after BDF administration (data not shown).

\section{NAC Prevents Early Hemoglobinuria but Does Not Affect Late Hematuria}

Based on our data that warfarin induces oxidative stress in the rat kidney [8], we tested the hypothesis that early BDF-associated hemoglobinuria could be due to oxidative stress. To test this, rats were given NAC per os in drinking water $24 \mathrm{~h}$ prior to the BDF administration. NAC decreased early hemoglobinuria associated with $0.4 \mathrm{mg} / \mathrm{kg}$ BDF in a dose-dependent manner. Pre-treatment with $10 \mathrm{mg} / \mathrm{kg} /$ day NAC partially decreased hemoglobinuria, whereas $100 \mathrm{mg} / \mathrm{kg} /$ day NAC completely prevented BDF-induced hemoglobinuria (fig. 3a). The decrease in hemoglobinuria was associated with a decrease in gross hemolysis and lesser changes in hematocrit associated with BDF poisoning (fig. 3b). In contrast, pre-treatment with NAC did not affect late hematuria caused by $0.4 \mathrm{mg} / \mathrm{kg}$ BDF at either dose (fig. 3c). Similarly, the BDF anticoagulation was not affected by NAC (data not shown), similarly to effects of NAC on warfarin anticoagulation [12].

\section{Discussion}

To the best of our knowledge, this is the first and only report that describes early renal effects of BDF. We demonstrated that BDF has dual action on the kidney: (1) it results in early transient hemoglobinuria and (2) it induces true glomerular hematuria, which is associated with anticoagulation. These early and late BDF effects on the kidney were dose-dependent. The late hematuria in experimental animals was demonstrated earlier by our group in rats who had free access to BDF in pellets ('uncontrolled' BDF) [9]. Patients with BDF intoxication also present with hematuria which is associated with coagulopathy $[13,14]$. However, the early transient hemoglobinuria is a novel finding, which may be a useful biomarker of BDF poisoning.

Based on our data, we propose that early effects of BDF are mediated via oxidative stress resulting in hemolysis, as is evident by gross hemolysis and decreased hematocrit after BDF administration. Indeed, earlier, we demonstrated increased oxidative stress in the kidneys in warfarin-treated rats [12]. It is likely that BDF also induces oxidative stress; such an effect is supported by our data showing that pre-treatment with NAC prevents BDF-induced early hemoglobinuria (fig. 3). The source of this oxidative stress is not yet known. One possibility is that oxidative stress is mediated by the bromine atom present in BDF. It is well known that bromobenzene is a potent mitochondrial inhibitor $[15,16]$; therefore, the bromobenzene moiety in BDF may exert similar effects. It is also possibly that the bromine group is ionized to a bromide ion that is released after the BDF absorption in the gastrointestinal tract (fig. 4), after that a conversion of bromide ion to nitrous bromide or hypobromous acid can occur, which is known to affect mitochondrial functions, resulting in increased oxidative stress [17]. It has been demonstrated that warfarin interacts with polymorphonuclear leukocyte myeloperoxidase [18], which may induce hemolysis. It is possible that BDF also may interact with polymorphonuclear leukocytes and thereby also induce rapid temporal hemolysis. Finally, the strong hydrophobic nature of BDF (its $\log \mathrm{p}$ value, a measure of relative solubility in octanol vs. water, is 8.2 , almost 5 orders of magnitude greater than that of warfarin, which is 2.8) raises the possibility that BDF directly interacts with cell membranes (either endothelial or hepatocytes), leading to cell damage. The BDF-induced hemolysis is rapid and temporal, as it is evident by rapid recovery of hematocrit within $24 \mathrm{~h}$ after the BDF treatment. Such rapid recovery of hematocrit is probably related to a large regenerative 


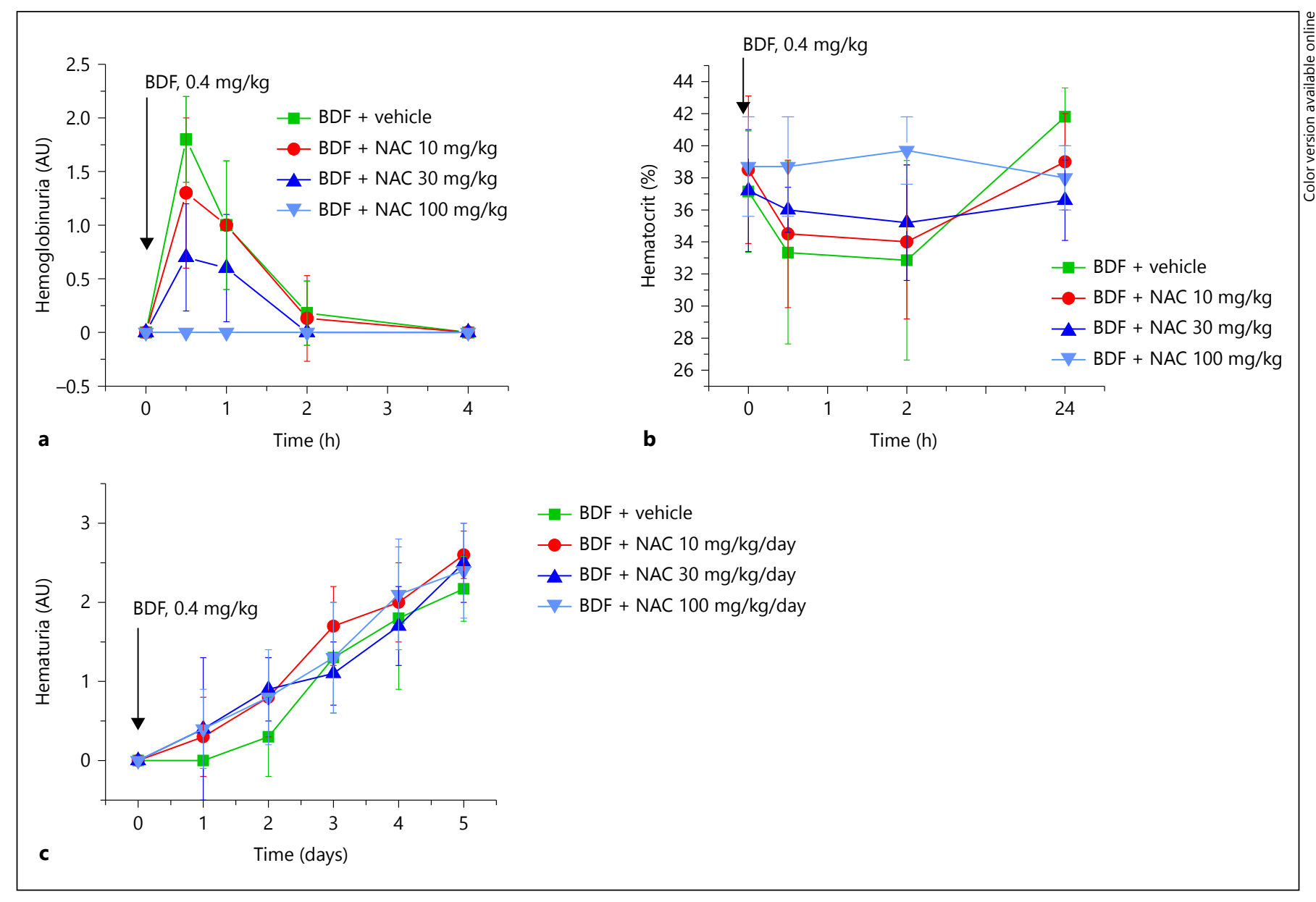

Fig. 3. Effects of pre-treatment with NAC on BDF-induced early hemoglobinuria and hematuria. The following number of animals was treated with $0.4 \mathrm{BDF}$ and NAC: $10 \mathrm{mg} / \mathrm{kg} /$ day, $\mathrm{n}=8 ; 30 \mathrm{mg} /$ $\mathrm{kg} /$ day, $\mathrm{n}=7 ; 100 \mathrm{mg} / \mathrm{kg} /$ day, $\mathrm{n}=9$; BDF-only rats, $\mathrm{n}=16$. a Rats were pre-treated with NAC for $24 \mathrm{~h}$ prior to $0.4 \mathrm{mg} / \mathrm{kg} \mathrm{BDF}$ administration in the doses shown. Treatment with NAC was continued at the same doses until the end of the studies. Hemoglobin-

capacity of the bone marrow and influx of RBCs from the extravascular storage [19-21].

In contrast to rapid actions, the delayed effects of BDF are more likely related to the vitamin $\mathrm{K}$ depletion and resulting excessive anticoagulation (fig. 4) [9]. As the case for warfarin [11], these effects are probably mediated through decreased thrombin activity, increased oxidative stress and endothelial cell injury with the consequent damage of the vascular barrier in multiple organs, including the kidney [9].

Based on this pathogenesis of BDF actions, we propose that early hemoglobinuria and delayed hematuria are novel biomarkers of BDF exposure and toxicity. Specifically, early transient hemoglobinuria indicates BDF in- uria was detected by DiaScreen reagent strip (dipstick). b Effects of NAC on BDF-induced changes in hematocrit. Rats were pretreated with NAC for $24 \mathrm{~h}$ prior to $0.4 \mathrm{mg} / \mathrm{kg} \mathrm{BDF}$ administration. c Effects of NAC on $0.4 \mathrm{mg} / \mathrm{kg}$ BDF-associated hematuria. Animals were treated with NAC for the duration of the studies in the doses shown.

gestion, whereas late hematuria indicates BDF-induced coagulopathy. These can be used as monitoring and treatment biomarkers in the case of incidental BDF ingestion or mass casualty associated with BDF poisoning (fig. 5). Monitoring of hematuria/hemoglobinuria is relatively simple, can be easily achieved in the outpatient setting; therefore, useful in a mass casualty scenario. We propose that after possible BDF ingestion, patients should be tested as soon as possible for hematuria (hemoglobinuria) by a dipstick (within the first $4-12 \mathrm{~h}$ ). Those who are positive should be retested again in 6-8 h. If the hematuria (hemoglobinuria) becomes negative, it is indicative of BDF poisoning (early transient hemoglobinuria as the biomarker). These patients should be monitored for coagu- 
Fig. 4. Proposed pathogenesis of BDF effects. BDF results in a transient hemolysis by increased oxidative stress after rapid absorption from the gastrointestinal tract. Later, BDF actions are related to vitamin $\mathrm{K}$ depletion and decreased thrombin activity. In patients who survived, long-term BDF effects lead to endothelial cell injury, chronic anticoagulation and damage of the vascular barrier in multiple organs. MGP = Matrix glutamate protein; GAS-6 = growth arrest-specific gene 6 .

Fig. 5. Proposed protocol for monitoring and treatment after possible BDF ingestion.
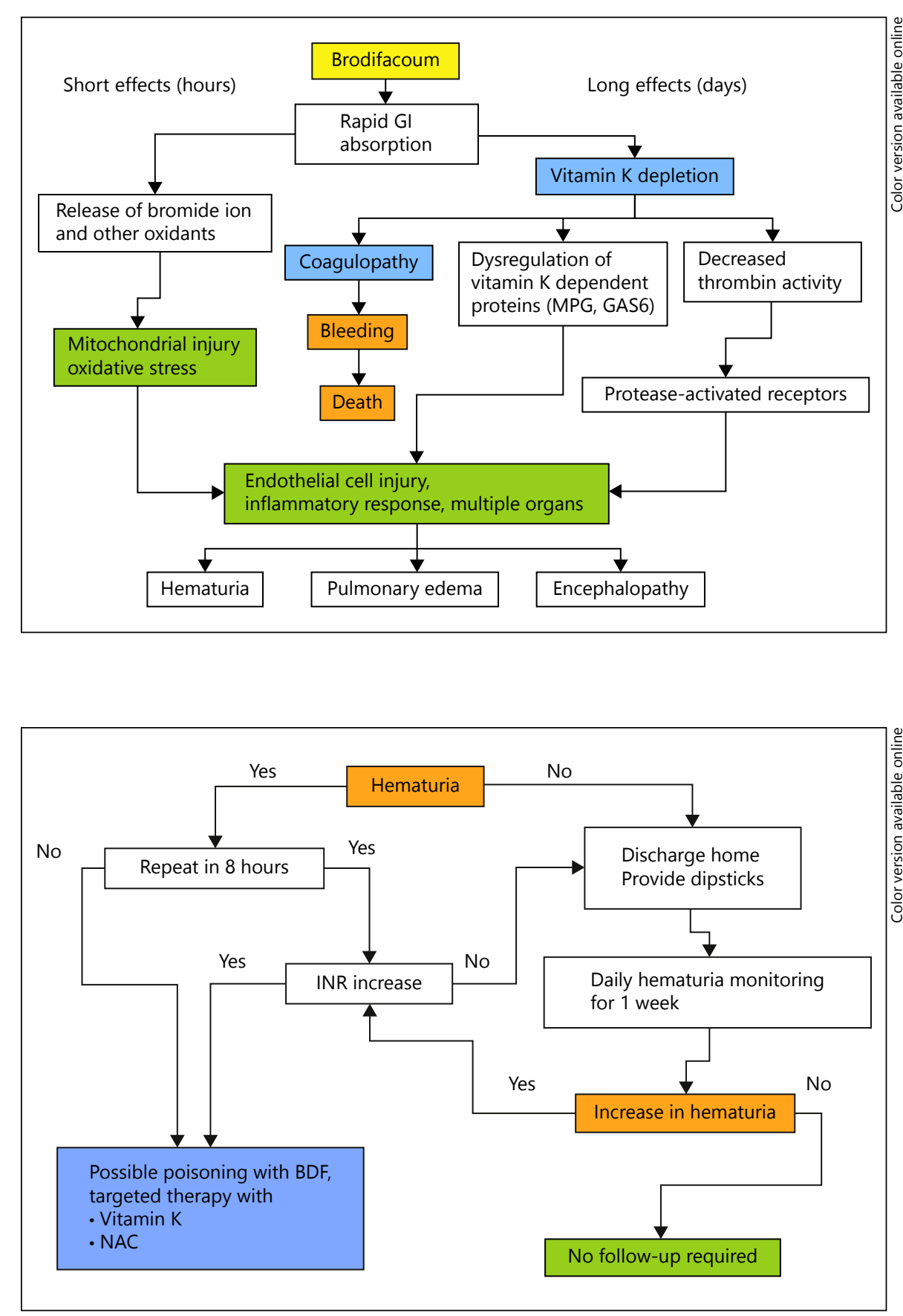

lopathy and treated, if needed. The main pathogenic mechanism of BDF is the depletion of bioavailable vita$\min \mathrm{K}$. Therefore, it is imperative to supplement vitamin $\mathrm{K}$ in patients with BDF poisoning. We believe that cotreatment with vitamin K and NAC will be beneficial, because vitamin $\mathrm{K}$ by itself may induce endothelial cell dysfunction via oxidative stress [22]. Importantly, NAC does not affect the late hematuria associated with BDF antico- agulation; therefore, early NAC administration will not mask hematuria in BDF poisoned patients (fig. 3c) [12]. Those who have persistent, but not increasing hematuria after $6-8 \mathrm{~h}$ of the initial testing likely have an underlying condition causing hematuria or BDF poisoning that occurred more than $24-48$ prior. These patients should be checked for coagulation (PT) in order to exclude BDFassociated late hematuria, and, if PT is normal, can be 
discharged with dipsticks provided to monitor daily hematuria for a week. For people with suspected BDF intoxication and with negative initial hematuria, the differential diagnosis includes the following: (1) no BDF exposure or (2) the test was performed too late, after hemoglobinuria spontaneously resolved. These patients may be released, but they should be provided with dipsticks and tested for hematuria daily. As an increase in hematuria is indicative of BDF poisoning, the patients who develop hematuria within a week after possible BDF poisoning should be immediately transferred to a medical facility, INR determined and treatment begun (fig. 5). Finally, our studies have only tested acute effects of BDF; however, several other superwarfarins are currently in use as rodenticides, and it is possible that the same rapid transient hemoglobinuria will occur following ingestion of those compounds as well.

In conclusion, we present evidences that BDF has dual action on the kidney, resulting in hemolysis and consecutive hemoglobinuria early after administration. These ef- fects are transient and diminish or disappear within $24 \mathrm{~h}$ after BDF poisoning. Late effects of BDF are anticoagulation related and include true glomerular hematuria with RBCs and dysmorphic RBCs in the urine. These novel biomarkers of BDF poisoning are potential biomarkers for the identification and triage of accidental or intentional BDF ingestion.

\section{Acknowledgment}

This study was supported, in a part, by the NIH grant U01 NS083457 (S.V.B., D.L.F., I.R. and G.W.), VA Merit Review Award (I.R. and G.W.), VA Research Career Scientist Award (D.L.F.) and start-up funds from the Department of Pathology, The Ohio State University (S.V.B.).

\section{Disclosure Statement}

The authors have no conflict of interest to disclosure.

\section{References}

1 Schulman S, Furie B: How I treat poisoning with vitamin $\mathrm{K}$ antagonists. Blood 2015;125: 438-442.

2 Bruno GR, Howland MA, McMeeking A, Hoffman RS: Long-acting anticoagulant overdose: brodifacoum kinetics and optimal vitamin K dosing. Ann Emerg Med 2000;36: 262-267.

3 Olmos V, López CM: Brodifacoum poisoning with toxicokinetic data. Clin Toxicol (Phila) 2007;45:487-489.

4 Hollinger BR, Pastoor TP: Case management and plasma half-life in a case of brodifacoum poisoning. Arch Intern Med 1993;153:19251928.

5 Patocka J, Petroianu G, Kuca K: Toxic potential of superwarfarin: brodifacoum. Mil Med Sci Lett 2013;82:9.

6 Vandenbroucke V, Bousquet-Melou A, De Backer P, Croubels S: Pharmacokinetics of eight anticoagulant rodenticides in mice after single oral administration. J Vet Pharmacol Ther 2008;31:437-445.

7 Chua JD, Friedenberg WR: Superwarfarin poisoning. Arch Intern Med 1998;158:19291932.

8 Mowry JB, Spyker DA, Cantilena LR, Jr, McMillan N, Ford M: 2013 annual report of the American association of poison control centers' national poison data system (NPDS): 31st annual report. Clin Toxicol (Phila) 2014; 52:1032-1283.

9 Ware K, Brodsky P, Satoskar AA, Nadasdy T, Nadasdy G, Wu H, Rovin BH, Bhatt U, Von
Visger J, Hebert LA, Brodsky SV: Warfarinrelated nephropathy modeled by nephron reduction and excessive anticoagulation. J Am Soc Nephrol 2011;22:1856-1862.

10 Brodsky SV, Satoskar A, Chen J, Nadasdy G, Eagen JW, Hamirani M, Hebert L, Calomeni E, Nadasdy T: Acute kidney injury during warfarin therapy associated with obstructive tubular red blood cell casts: a report of 9 cases. Am J Kidney Dis 2009;54:1121-1126.

11 Ozcan A, Ware K, Calomeni E, Nadasdy T, Forbes R, Satoskar AA, Nadasdy G, Rovin BH, Hebert LA, Brodsky SV: 5/6 nephrectomy as a validated rat model mimicking human warfarin-related nephropathy. Am J Nephrol 2012;35:356-364.

12 Ware K, Qamri Z, Ozcan A, Satoskar AA, Nadasdy G, Rovin BH, Hebert LA, Nadasdy T, Brodsky SV: N-acetylcysteine ameliorates acute kidney injury but not glomerular hemorrhage in an animal model of warfarin-related nephropathy. Am J Physiol Renal Physiol 2013;304:F1421-F1427.

13 Wu YF, Chang CS, Chung CY, Lin HY, Wang CC, Shen MC: Superwarfarin intoxication: hematuria is a major clinical manifestation. Int J Hematol 2009;90:170-173.

14 Yu HY, Lin JL, Fu JF, Lin JH, Liu SH, Weng $\mathrm{CH}$, Huang $\mathrm{WH}$, Chen $\mathrm{KH}$, Hsu CW, Yen $\mathrm{TH}$ : Outcomes of patients with rodenticide poisoning at a far east poison center. SpringerPlus 2013;2:505.

15 Schnellmann RG, Ewell FP, Sgambati M, Mandel LJ: Mitochondrial toxicity of 2-bromohy- droquinone in rabbit renal proximal tubules. Toxicol Appl Pharmacol 1987;90:420-426.

16 Maellaro E, Del Bello B, Casini AF, Comporti M, Ceccarelli D, Muscatello U, Masini A: Early mitochondrial disfunction in bromobenzene treated mice: a possible factor of liver injury. Biochem Pharmacol 1990;40:1491-1497.

17 Blondin GA, Green DE: The mechanism of mitochondrial swelling. V. Permeability of mitochondria to alkali metal salts of strong acid anions. J Bioenerg 1970;1:193-213.

18 Belij S, Miljković D, Popov A, Subota V, Timotijević G, Slavić M, Mirkov I, Kataranovski D, Kataranovski M: Effects of subacute oral warfarin administration on peripheral blood granulocytes in rats. Food Chem Toxicol 2012;50:1499-1507.

19 Connett RJ, Pearce FJ, Drucker WR: Scaling of physiological responses: a new approach for hemorrhagic shock. Am J Physiol 1986; 250(6 pt 2):R951-R959.

20 Glick YA, Wilson LD, Aiello J: Hematocrit and metabolic changes caused by varied resuscitation strategies in a canine model of hemorrhagic shock. Am J Emerg Med 2002; 20:303-309.

21 Streeton JA: Traumatic haemogloinuria caused by karate exercises. Lancet 1967;2:191-192.

22 Tirapelli CR, De Andrade CR, Lieberman M, Laurindo FR, De Souza HP, de Oliveira AM: Vitamin K1 (phylloquinone) induces vascular endothelial dysfunction: role of oxidative stress. Toxicol Appl Pharmacol 2006;213:1017. 